\title{
Utilization of Eddy Current Tomography in Automotive Industry
}

\author{
P. NowAK ${ }^{a, *}$, M. NOWICKI ${ }^{a}$, A. JUŚ ${ }^{b}$ AND R. SzEWCZYK ${ }^{a}$ \\ ${ }^{a}$ Institute of Metrology and Biomedical Engineering, Warsaw University of Technology, \\ sw. A. Boboli 8, 02-525 Warsaw, Poland
}

${ }^{b}$ Industrial Research Institute for Automation and Measurements, Al. Jerozolimskie 202, 02-486 Warsaw, Poland

\begin{abstract}
Paper presents utilized innovative setup for eddy current tomography and possibility of its utilization in automotive industry. Described tomography setup is designed for testing axisymmetric objects thus motor valve was selected for exemplary testing. Tests were conducted on motor valve in original state. Afterwards reference defect was created on element and measurements were repeated. Significant difference between tests results were observed, thus potential for utilization in automotive industry was confirmed. Finite element method simulations were applied in order to confirm the measurement results. Calculations were conducted in open-source finite element method software, which solves Maxwell equations in the A-V form. Modelling results confirm possibility of finite element method-based inverse tomography transformation.
\end{abstract}

DOI: 10.12693/APhysPolA.131.1168

PACS/topics: 85.80.Jm, 87.10.Kn

\section{Introduction}

Tomography tests are one of the most intensively developed form of non-destructive testing of many mechanical elements. The main advantage of tomography is the unique possibility of obtaining data about both shape and the nature of discontinuities in the tested element. Commonly used X-ray tomography is a source of many risks typical for ionizing radiation techniques. This results in significant cost and difficulties for usage in industrial environment, and may sometimes be dangerous for operators.

An alternative can be eddy current tomography [1]. It allows for parallel measurements of resistivity and magnetic susceptibility of material in the element tested [2]. This results with new possibilities for detection of discontinuities in structures while performing tests in industrial conditions.

\section{Tomography measurements}

Tests were conducted on an eddy-current tomography setup [3], where tested element is moved linearly between the driving coil and the measuring coil. Additionally, in each linear position object is rotated. Sine current from the generator powers driving coil, which induces sine magnetic field. Variable magnetic field induces eddy currents in the tested element. Depending on tested element material, conductivity and permeability, changed signal is sensed by measuring coil. After proper signal conditioning, data about amplitude and phase shift (respectively to the driving coil signal) are measured.

*corresponding author; e-mail: p.nowak@mchtr.pw.edu.pl
Due to particular design of the measurement setup (for testing axisymmetric objects), a typical motor valve was selected. 3D model of utilized test stand, with the tested valve, is presented in Fig. 1.

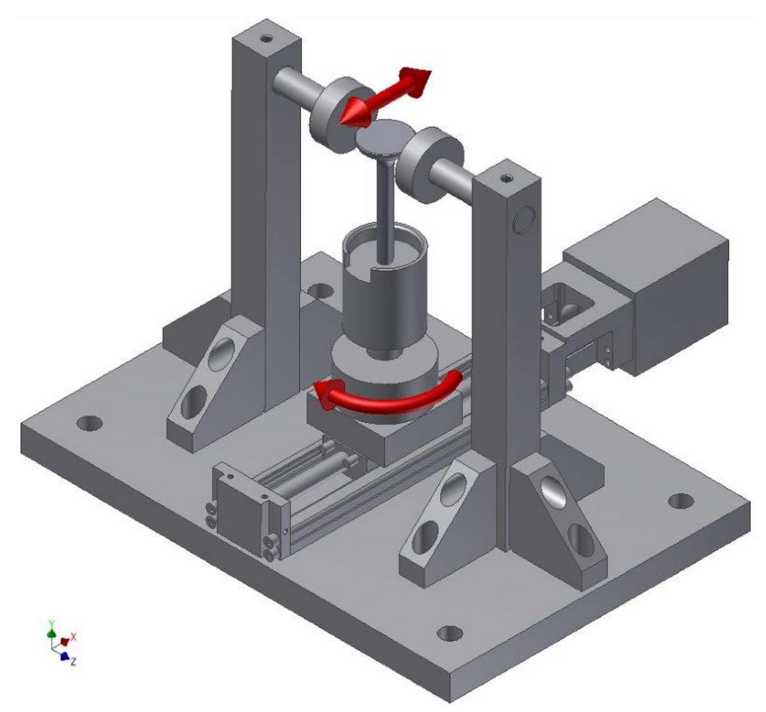

Fig. 1. 3D model of utilized test stand with arrows marking the movement of tested object.

Tested object was measured on the setup firstly in the delivery condition and secondly after making a reference defect (longitudinal cut on the edge of valve). Obtained results seemed similar, but their differential analysis proved noticeable influence of the defect on the results. Results of differential analysis are presented in Fig. 2.

In the top graph an amplitude difference is presented, whereas bottom graph shows difference in the phase shift. Each $X Y$ point in the graph corresponds to single measurement position and the color of the point ( $Z$ axis projected onto the graph plane) represents the linearly nor- 

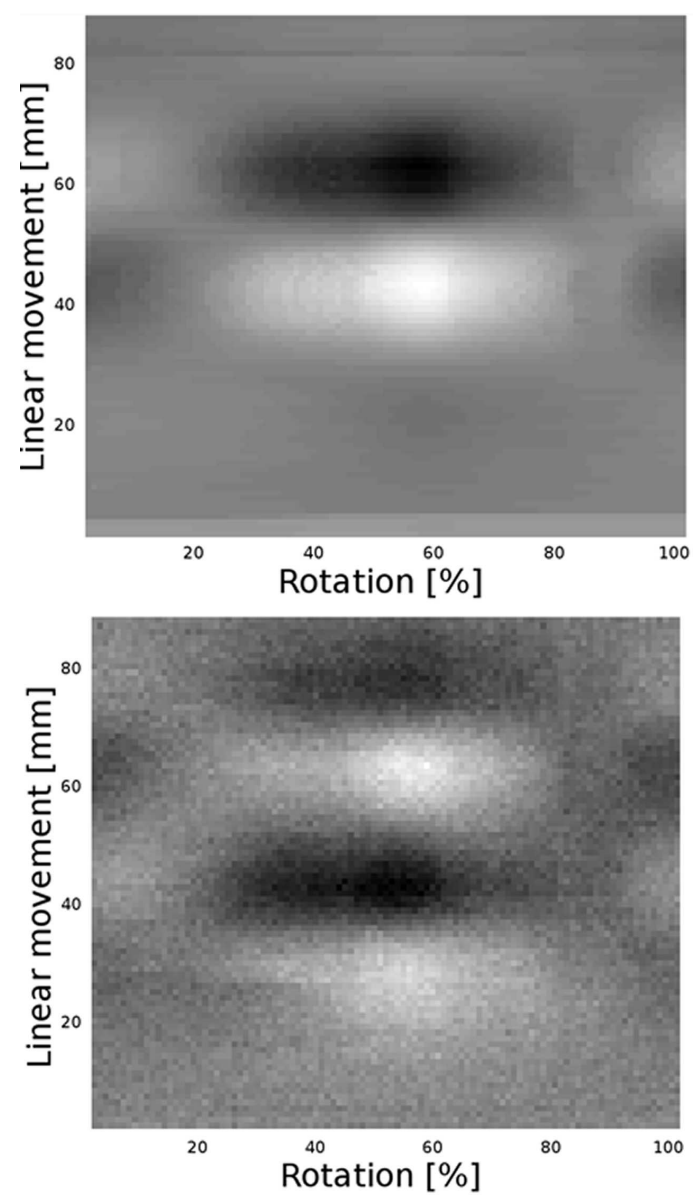

Fig. 2. Results of differential analysis of the tomography measurement results on the reference sample.

malized value of the analyzed difference in corresponding measurement points.

\section{FEM modeling results}

In order to confirm influence of the reference defect on the measurement result and to present possibility of inverse tomography transformation, a finite element method (FEM) modeling of the simplified problem was conducted. Simulations were made in the open-source ElmerFEM software, which utilizes first order Whitney elements for solving Maxwell equations in frequency domain $[4,5]$. Scalar values of potential are calculated by the Lagrange interpolation. Due to utilization of edge, the Whitney edge elements solver is suitable only for three-dimensional problems.

Solver is based on magnetostatic Maxwell equations which describe the dependences between magnetic and electric field and also the properties of the magnetic field. Other equations utilized in solver are the Gauss law and the Faraday law.

Tomography setup was simplified to the two coil setup with tested element between them. Exciting coil was modeled as a single turn coil, whereas measurement coil was simplified as a constant disk for better representation of integration of magnetic field phenomena. Whole setup was placed in a large modeling sphere (10 times bigger than modelled objects), which serves two purposes during modelling. Firstly, sphere provides place for modelling of distribution of magnetic field in the air around the objects. Secondly, external surface of the sphere is utilized for applying proper Dirichlet boundary condition for the differential equation solvers.

Simplified geometry model was meshed in open-source meshing software - Netgen 5.3. Exemplary view of meshed model is presented in Fig. 3. Measurement coil (placed to the right of the valve model) is uniformly meshed. Mesh density in tested element significantly increases closer to the reference defect due to the Delaunay algorithm. Also the exciting coils' mesh density increases closing to the valve model. In the background external surface of the modeling sphere is presented as well.

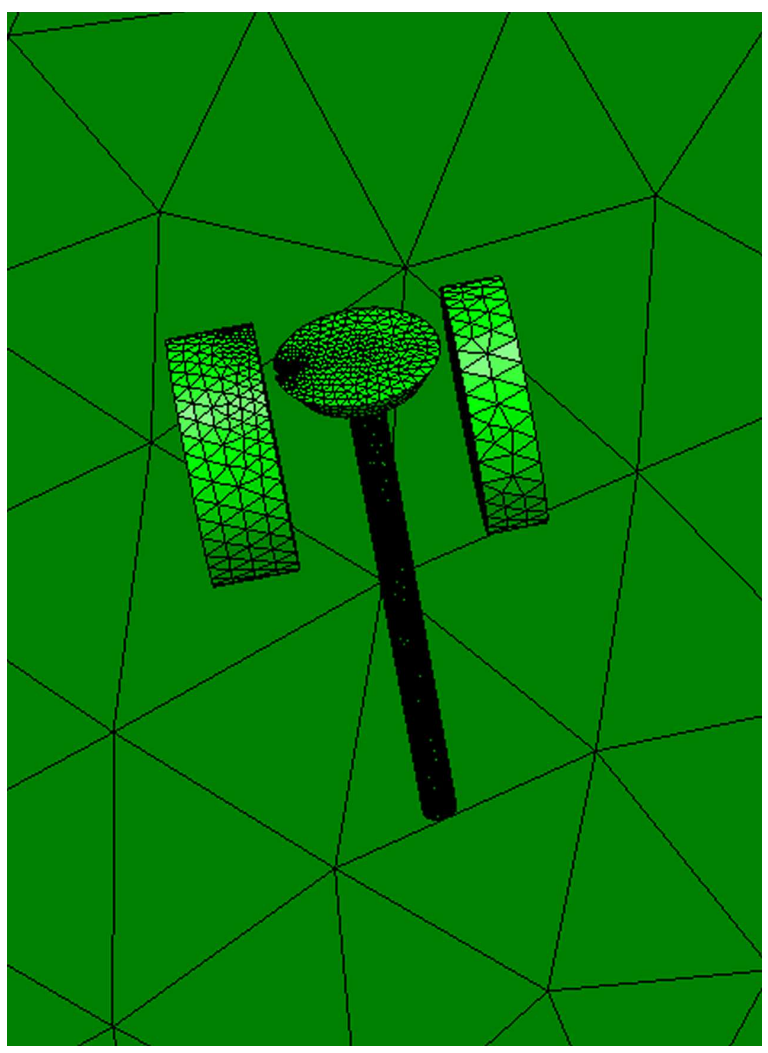

Fig. 3. View of the meshed model of tomography setup.

Simulations were conducted in a single position of the measured object, when valve main axis is perpendicular to the axis of the coils. Accordingly to the real measurements simulations were made on the whole valve model and on the model with reference damage. During simulations exciting coil was powered with alternate current, whereas the magnetic potential value on the external surface of the modelling sphere was forced 0 (the Dirichlet condition).

Results of the eddy-current distribution modelling is presented in Fig. 4. Higher density of the current vec- 

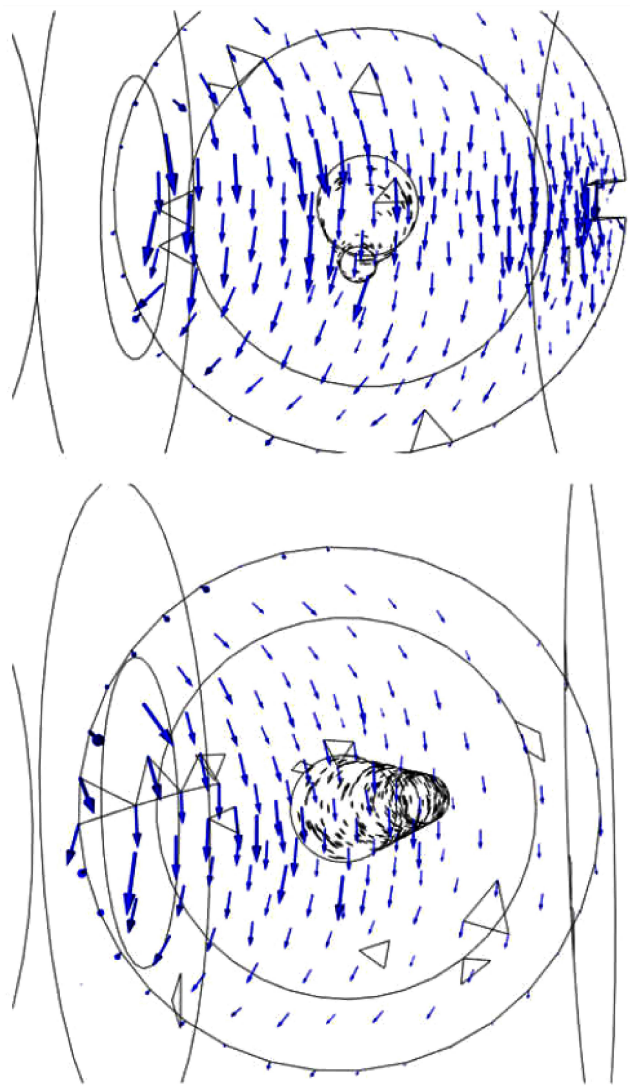

Fig. 4. Vectors of eddy currents induced in the top layer of the tested element on the sample with (top) and without (bottom) reference defect.

tors in the top image is caused by higher mesh density. Disturbance of current flow, caused by elements defect is clearly visible. Basing on the forward modeling, inverse tomography transformation may be developed.

During typical production-line eddy currents tests, defective units are being removed. Due to proper reconstruction of tested element shape, basing on the tomography measurements, failure in production process can be detected.

\section{Conclusions}

Innovative method for testing commonly produced element was developed as presented. Measurements were conducted on typical engine valve with and without reference defect. Differential analysis of the measurement results, confirm influence of the objects structure on the obtained results which was also confirmed with FEM analysis.

Further research shall concern development of inverse tomography transformation, which will lead to proper and reliable reconstruction of the elements shape. Other way may be preparation of reference results database for different types of objects and theirs defects.

\section{Acknowledgments}

This work was partially supported by the statutory founds of Institute of Metrology and Biomedical Engineering, Warsaw University of Technology (Poland).

\section{References}

[1] D. Premel, A. Mohammad-Djafari, IEEE Trans. Magn. 31, 2000 (1995).

[2] M. Soleimani, J. Electromagnet. Wave 23, 785 (2009).

[3] J. Salach, Jamris 8, 11 (2014).

[4] R. Szewczyk, J. Salach, J. Ruokolainen, P. Raback, M. Nowicki, in: Proc. 20 th Int. Conf. on Applied Physics of Condensed Matter (APCOM2014), Eds. J. Vajda, I. Jamnicky, 2014, p. 183.

[5] A. Bossavit, IEE Proc. A 135, 493 (1988). 